\title{
MECHATRONICS ENABLING KIT FOR 3D PRINTED HAND PROSTHESIS
}

\author{
Wong, Tat Hang (1); Asnaghi, Davide (2); Leung, Suk Wai Winnie (1) \\ 1: Division of Integrative Systems and Design, Hong Kong University of Science and Technology; 2: \\ Department of Bioengineering, University of California Berkeley
}

\begin{abstract}
New advances in both neurosciences and computational approaches have changed the landscapes for smart devices design serving mobility-related disabilities. In this paper we present the integration of affordable robotics and wearable sensors through our mechatronic product platform, Sparthan, to enable accessibility of the technology in both the power prosthesis and neurorehabilitation space. Sparthan leverages 3rd party EMG sensors, Myo armband, to process muscles sensor data and translate user intention into hand movements. Key innovation includes the modularity, scalability and high degree of customization the solution affords to the target users. User-centered design approaches and mechatronic system design are detailed to demonstrate the versatility of integrative systems and design. What started off as an engineering research endeavor is also positioned to be deployed to deliver real-world impact, especially for prosthesis users in developing countries.
\end{abstract}

Keywords: Mechatronics, 3D printing, Lean design, User centred design, Integrated product development

Contact:

Leung, Suk Wai Winnie

Hong Kong University of Science and Technology

Division of Integrative Systems and Design

Hong Kong S.A.R. (China)

eewswleung@ust.hk

Cite this article: Wong, T.H., Asnaghi, D., Leung, S.W.W. (2019) 'Mechatronics Enabling Kit for 3D Printed Hand Prosthesis', in Proceedings of the 22nd International Conference on Engineering Design (ICED19), Delft, The Netherlands, 5-8 August 2019. DOI:10.1017/dsi.2019.81 


\section{INTRODUCTION AND MOTIVATION}

New advances in both neurosciences and computational approaches have changed the landscapes for smart devices design serving mobility-related disabilities. In this paper we will look at how we integrated affordable robotics and wearable sensors through our mechatronic product platform to enable accessibility of the technology in both the power prosthesis and neurorehabilitation space.

While we believe that our solution will benefit most for those in developing countries supported by the wider maker's community, the project started from a humble student-driven innovation initiative in HKUST in Hong Kong. Sparthan uses a third party EMG sensors, the Myo Armband, to measure electrical activity from muscles to detect gestures made by one's hands. Using a 9-axis IMU, it also senses the motion, orientation and rotation of the forearm. Raw EMG and IMU data are the streamed for on-board processing to drive the motorized digits.

The initial target users will be general needs for hand prosthesis in developing countries, and serving globally children who have lost partial or complete functionality of their hand. Some statistics on the target users:

- More than 40,000 children are born with congenital upper limb defects every year.

- There are 3.5 million upper limb amputees worldwide, $80 \%$ of them in developing countries.

- 56 million elderlies over 80 years old suffer from (muscle degeneration).

On the market, powered prosthesis with enabling basic motions are very expensive, starting from USD1000. These solutions are limiting for children with the lengthy fitting process and how quickly the prosthesis are outgrown. Market research shows a gap in the market for a solution that provides affordable and accessible prosthesis; existing solutions and market gap will be discussed in Section 2. Low cost and accessibility, however, are not sufficient to create meaningful impact in a scalable and adaptable fashion. Within this project, we employ lean product development cycle, machine learning for gesture recognition, and adaptive model-based control to deploy the first version of Sparthan.

\section{SYSTEM-LEVEL DISSECTION OF EXISTING SOLUTIONS}

In response to the challenges, shortcomings, and high cost of traditional prostheses multiple organizations started to pioneer new and innovative ways to manufacture cheaper prosthetic hands.

\section{1 e-Nable community}

Arguably the most influential non-profit in the prosthetic hands space, e-Nable describes itself as a global network of volunteers who are using their 3D printers, design skills, and personal time to create free 3D printed prosthetic hands for those in need - with the goal of providing them to underserved populations around the world.

e-Nable was started in 2013 by a small group of committed individuals offering their 3D printers for manufacturing cheaper, mechanical prosthetic hands, inspired by the work of Ivan Owen. The organization is now the only provider of 3D printed prosthetic hands with a global network completely run by volunteers, offering 4 different customizable hand models and full support on the build process. The mission of the e-Nable community is to provide access to prosthetic hands inside communities that would otherwise not have any other means of doing so. Their devices are not aimed at replacing traditional professionally made prostheses, but at creating an extremely low cost solution that can be beneficial to specific section of the population (i.e. children, patients in developing countries, etc.)

\subsection{Million Hands project}

The Million Hands project is an initiative started by the BEST Lab at Berkeley, overseen by Ph.D. candidate Daniel Lim. Million Hand is specifically aimed at lowering the cost of deploying 3D printed prostheses, by engineering better technologies to design and manufacture devices at a large scale. Million Hands has won numerous awards including Million Hands: Prosthetic Hands for Children through an Open Source Platform, 3D Printers and Sensors and Helping Hands - Playground Edition. While the e-Nable community is focused on creating and maintaining a network of volunteer around the world, Million Hands is mostly working on improving the underlying technologies in the field to support and complement deployment in the future. The University of California Berkeley is also actively involved in the project, supporting the initiative and expanding its scope. 


\subsection{Open Bionics}

Open Bionics is a company based in the United Kingdom, funded by Joel Gibbard in 2014. Joel started working on open source and open hardware prosthetic hands under the Open Hand project. The project, started in 2013, was aimed at creating and publishing mechanical designs for a 3D printable prosthetic hand that would be cheaper and more customizable than traditional equivalents. After publishing initial designs, the Open Hand project morphed into a full-fledged company, under the name Open Bionics.

Open Bionics has been the first commercial company to get regulatory approval for using a 3D printed prosthetic arm as a medical device. The main strategy of the company is using the same underlying platform, named Hero Arm, as the functional base of every prostheses, customizing the external appearance through the use of skins and 3D printed covers. Open Bionics has managed to bridge the gap between the open source community and the professional prosthetic market, bringing regulatory approval to 3D printed prosthetics in an unprecedented market shift. Their operations, however, are currently limited to the United Kingdom, since the prostheses are supplied and regulated by the \{NHS\}, a national institution. Despite this limitation, Open Bionics provides open source designs both for mechanics and electronics in their devices, allowing anyone to improve and continue their work in other countries.

The above solutions all have respective strength merits but there exists a gap in the prosthesis market space that our solution addresses.

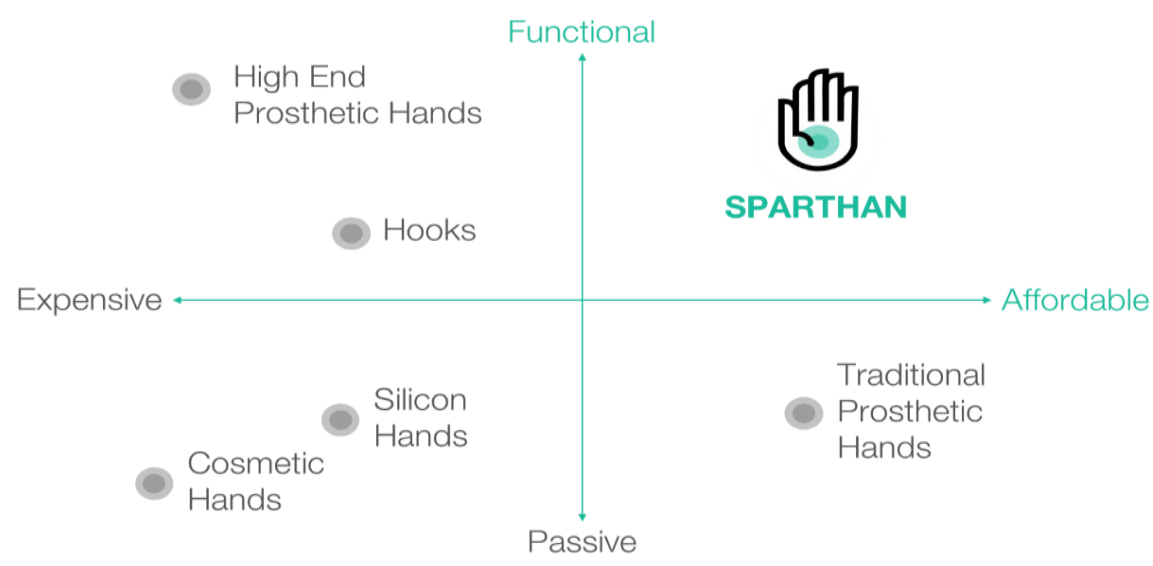

Figure 1. Market Gap filled by Sparthan

\section{USERS' NEEDS AND REQUIREMENTS: MODULARITY, SCALABILITY AND HMI}

Market research was performed in Hong Kong, Seoul, Beijing and San Francisco Bay Area. Stakeholders including potential users, family members, hospital staffs and local maker community were surveyed and interviewed. Some interesting findings were uncovered to show that beyond simple functionalities offered by prosthesis, the mental benefit of feeling empowered and included by their peers is considered important. Most feels powerless with the disability that holds them back from job and financial security.

The high level needs deduced from the primary market research ranked in order of importance include:

a. Weight of the product

b. Functionality of the product

c. Durability of the product

d. Appearance of the product

Using Kano model and simplified QFD, three important requirements were further extrapolated. 


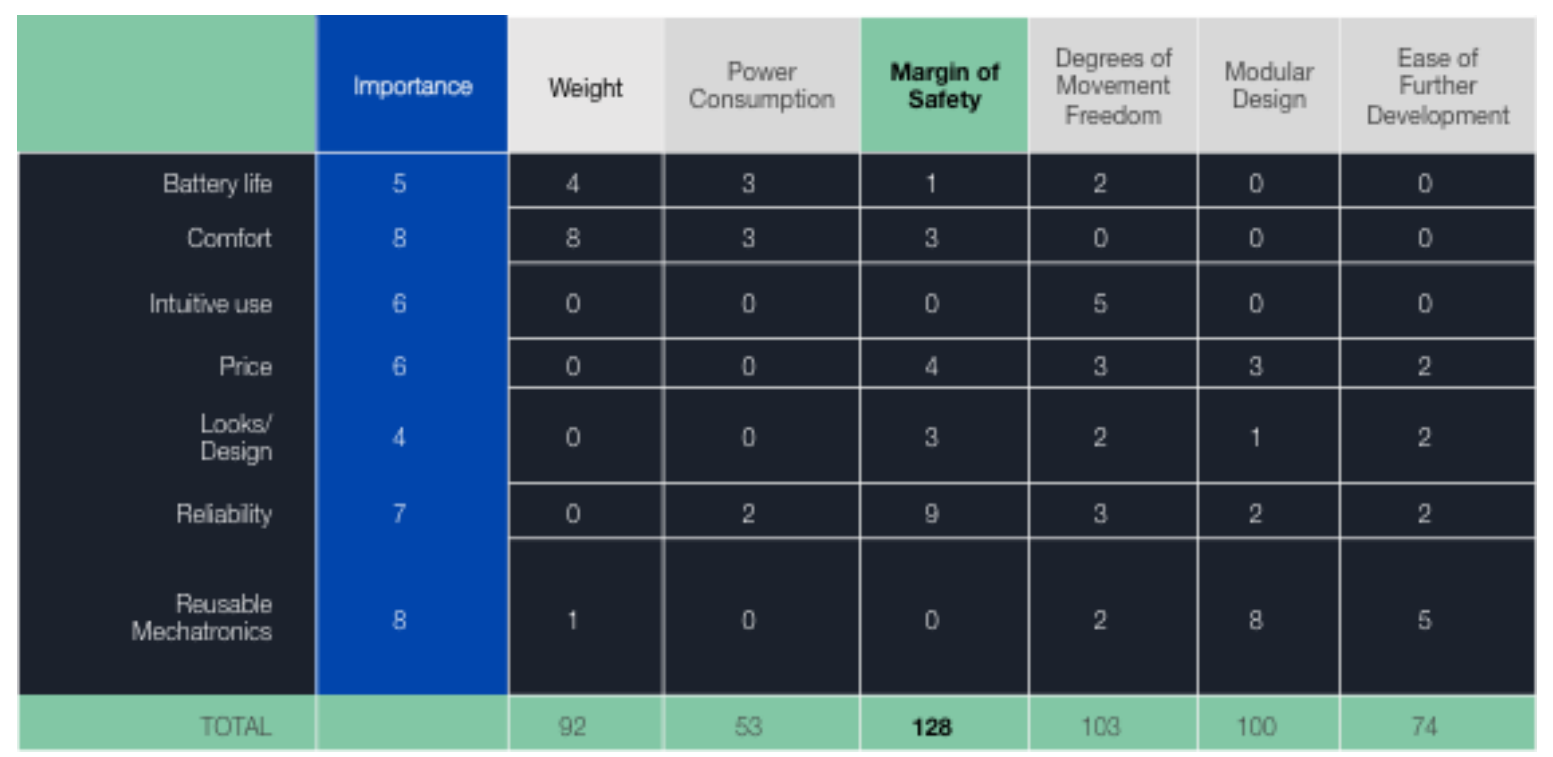

Figure 2. Simplified QFD prioritization of Sparthan's Product Features

Based on the QFD analysis, the following system requirements are determined.

\section{Table 1. High-level system requirements}

Bluetooth connectivity with The hardware platform must contain a Bluetooth wireless EMG armband transceiver to communicate with external EMG sensing hardware.

Positional control for At least 5 motors with close-loop positional control is required to individual fingers perform desired gestures under external loads.

Ease of further development The hardware platform should be able to be programmed in a high from the user community level programming language to encourage further development

Responsive human-machine Adequate processing power is required to run classification interaction

Ease of assembly through Because our solution relies on the local maker's resources and modularity support, the solution should be accessible and flexible.

Scalability for different Mechatronics modules should be compartmentalized in small form prosthesis' sizes factors to accommodate different 3D hand models.

A repeated painpoint voiced by the surveyed users is the discomfort of using the prosthesis due to illfitting and time-consuming, poor user-experience of the fitting procedure itself. While making our product accessible to the end-users give them more control over the fitting procedure, we are also placing ease-of-use design requirements on Sparthan to ensure

- Compatibility with existing 3D hands models by providing multiple versions

- Easy installation procedure for the makers with snap on mechanism and video tutorials

- Intuitive UI interface for app-based DIY process such as 3D scan, gesture mapping, etc.

The ability to incorporate 3rd party solution to process muscles sensor data and translate user intention into hand movements would only generate impact if we can effectively offer customization and advanced control capability; which would be described in the next section.

\section{SYSTEMS DESIGN - METHODOLOGIES AND SUBSYSTEMS DESIGN}

Creating a module that could interact with existing 3D models was the key to improve their functionality, without changing the dynamics of the open source community. For this reason Sparthan is compatible with all models based on flexible joints printing. This allows makers to access a wide range of resources on assembly and printing of the models, streamlining the makers' experience.

To provide an intuitive user interface we decided to use EMG signals coming from the patient's forearm. Muscles and tendons are still present in the forearm either in the event of an amputation or in the case of a congenital malformation. These leftover muscles can be contracted and controlled directly by the patient, providing an intuitive and fast way to interact with the prosthetic hand. 
A micro controller unit in the palm can then process the signals and detect when a particular gesture is being performed, actuating the corresponding fingers through the use of micro electric motors embedded in the palm.

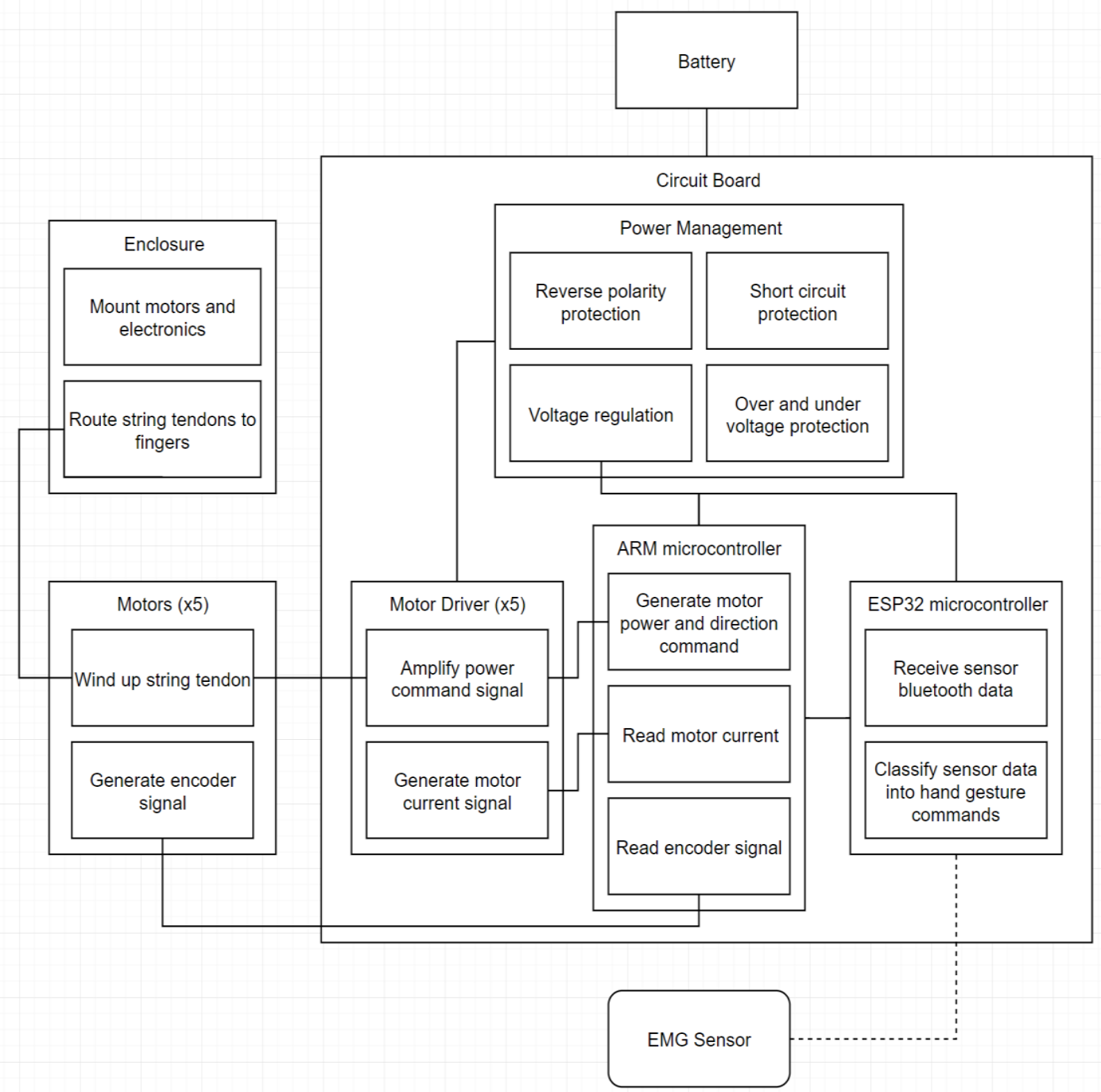

Figure 3. Sparthan's Overall System Diagram

\subsection{Mechanical}

Mechanical design of this project is based on user's need on modularity and scalability. The stringent requirement of fitting in a small form factor of a child's prosthesis drives the design towards small compartments and strategic layout of motor arrangements. In a Sparthan-equipped prosthetic hand, the two main sections of the mechanical system are:

\subsubsection{Sparthan module}

The Sparthan module enables 3d printed prosthetic hands to be controlled myoelectrically. It houses the Sparthan controller board and five geared DC motors securely. Attached to each geared motor shaft is a roller which pulls or releases a piece of string when the motor spins, allowing the string to act as tendon controlling the movement of prosthetic fingers. Hollow paths routed through the module housing guides the piece of string to the $3 \mathrm{~d}$ printed prosthetic hand. Additional slots and holes are present in the module housing to allow routing of power and data cables.

The Sparthan module housing is designed for manufacturing. To ensure the Sparthan module can be scalable to meet potential high demand, and varying manufacturing capabilities of makers across the world, the module housing can be produced by either stacking laser cut plastic sheets or 3D printed.

When choosing the housing material, the following requirements were considered: 
- Maximum working temperature has to withstand the high temperatures generated by the geared motors.

- The material has to be available in sheet form for laser cutting and in filament form for FDM $3 \mathrm{~d}$ printing.

- The material has to be durable and impact resistant for rough daily life use.

Driven by the above requirements, polycarbonate (PC) was chosen as the housing material.

\subsubsection{Prosthetic hand}

The Sparthan module is designed in a way such that it can be easily fitted into 3D printable prosthetic hand models with simple modifications. Using an additive manufacturing method such as 3D printing presents multiple advantages for designing prosthetic devices, especially with regards to customization. Comfort and fit are key factors for any prosthesis, and due to the high degree of customization required it is inherently hard to mass manufacture these devices with traditional methods. 3D printing, concluded by Jelle et al., provides a cost effective, customizable solution for designs that need to conform to the user's body shape as closely as possible, in particular for upper limb prostheses. A 3D printable Sparthan-ready prosthetic hand model is produced by boolean subtracting the Sparthan module from the original hand model, followed by cutting string paths that join the Sparthan module and fingers. Due to its close resemblance to a human hand, the Flexy Hand 2 prosthetic hand model was chosen as the basis for the first Sparthan-ready prosthetic hand.

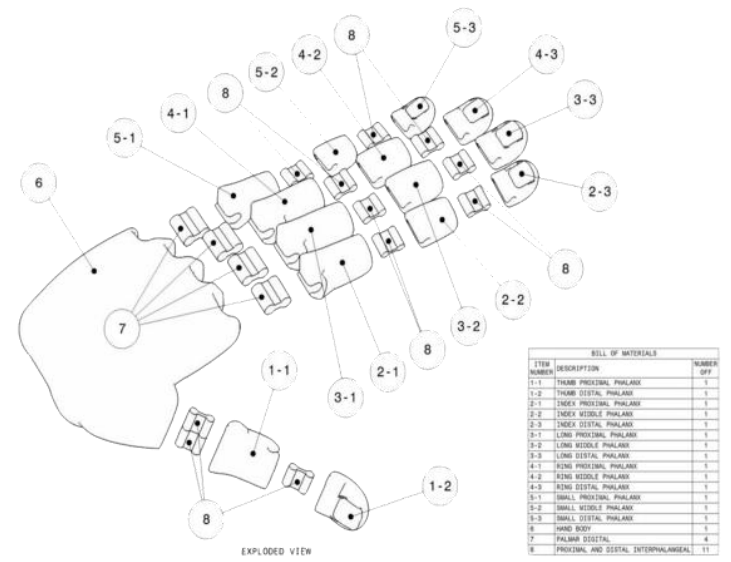

This particular 3D printable hand combines a very anthropomorphic design with good functional capabilities. The joints in the fingers are printed using flexible filament (e.g. TPU) which allows the fingers to spring back into position after being flexed by the motors.

Potentially, any design which shares this feature is compatible with our module, allowing users to choose and modify existing designs to be used with it.

Figure 4. Flexy Hand 2

Parametric equations in SolidWorks will be used in the future to perform dimensional analysis, and to simplify the model-based design. Relationships can be defined in the palm and fingers. The palm dimensions can first be defined, while the fingers' relations are uniquely attributed in relation to the palm. With these being identified, the hand will scale for different users using physical measurement fittings; allowing users from all ages with various physical attributes to adopt Sparthan.

\subsection{Electronics}

One of the key differentiating features of Sparthan consists in its ability to electronically control the finger movement through the use of motors. Motorized control is indeed what distinguishes a normal 3D printed prosthetic device from one equipped with Sparthan, and for this reason particular care was taken during the corresponding design process.

The two main functions of the electronics inside Sparthan can be described as:

\subsubsection{EMG recording}

The signal coming from the muscles needs to be sensed through differential electrodes applied to the skin and amplified with analog circuitry, before being processed to infer the patient's desired gesture. \{EMG\} signal classification is by itself a very hard task, strongly dependent on the quality of the data recorded as well as on the kind of classification algorithm chosen. For this reason we decided to employ a commercially available device that could record eight different signals all around the forearm and transmit them over BLE to the microcontroller in the hand. This device is the Myo 
armband by Thalmic Labs. This device not only offers high end analog circuitry, but also a custom API for Bluetooth communication, which allows for a very robust connection and lasting battery life.

There exists multiple brands for EMG armbands in the market, and each variation of EMG armband requires different wireless communication protocol and signal processing techniques. The Sparthan software platform was developed in a modular manner, such that variant specific armband communication and signal processing software modules were decoupled from the rest of the software platform. Modular software design allows for easy swapping of EMG sensing hardware, depending on device availability. At the time of writing, the MYO armband from Thalmic Labs and the GFORCE armband from Oymotion are

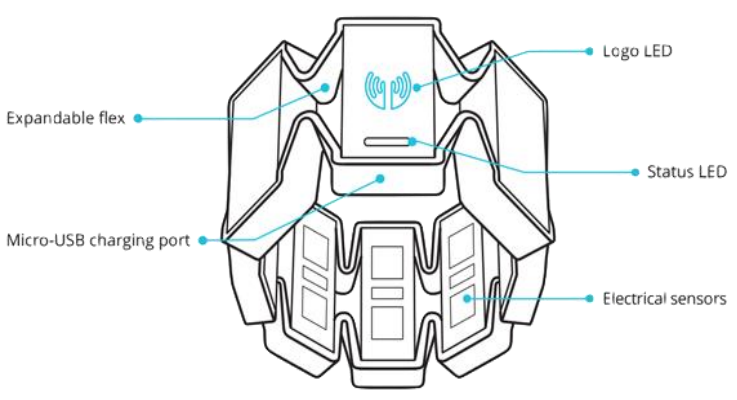

Figure 5. Myo armband supported.

\subsubsection{EMG decoding and actuation}

In order to process and decode the signal we designed a custom PCB capable of receiving the data through a Bluetooth module and process it. Designing a custom board [FIGURE: BOARD] allowed us to reduce the components' footprint, while using high end motor drivers directly connected to the microcontroller. The motor drivers we selected present two important features that help to reduce power consumption.

a. Motor current sensing

b. Over-current protection

Sensing the current to each individual motor allows the microcontroller to know exactly when the finger is touching the object and the motor is stalling, thanks to the peak in current draw. This peak is limited by the over-current protection and can be used to detect exactly when to stop the motor's motion. The board also provides solder points for the motors, allowing for a direct connection that greatly simplifies cable management. Each motor attachment point has six pins, two for power and four for the Hall Effect magnetic sensor that act as encoders for the motor's rotation.

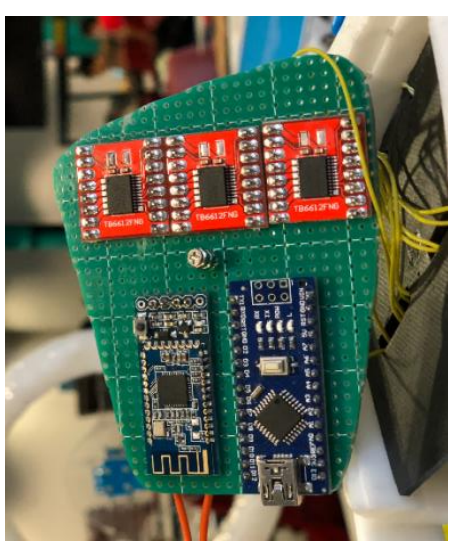

Figure 6. (Left) 1st generation mainboard,
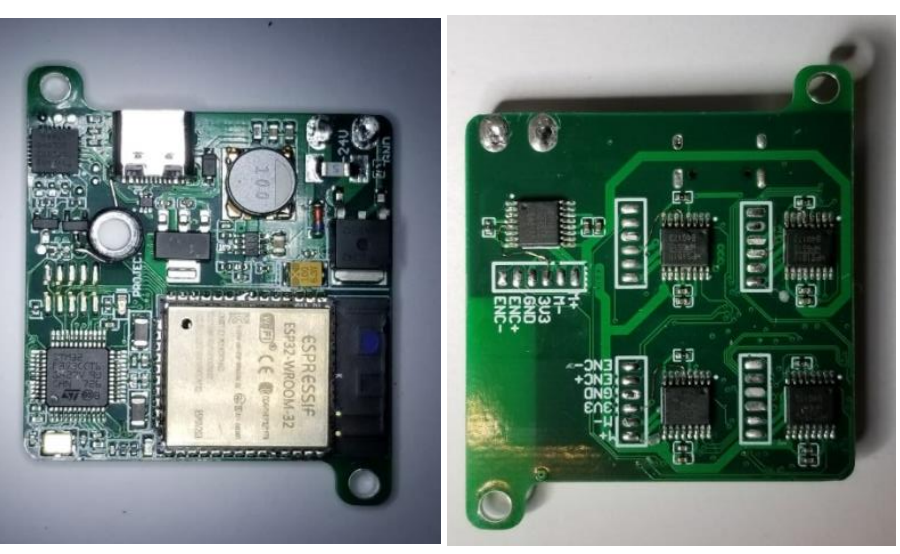

drives

\subsection{Adaptive model-based control}

During the proof-of-concept first generation prototype, attention was placed on gesture mapping by running classification algorithms to estimate user intended gestures. Understanding that simple classifiers such as regression or SVM would not offer the desired accuracy, deep learning classifiers are built to optimize high-level motion control for the prosthesis 


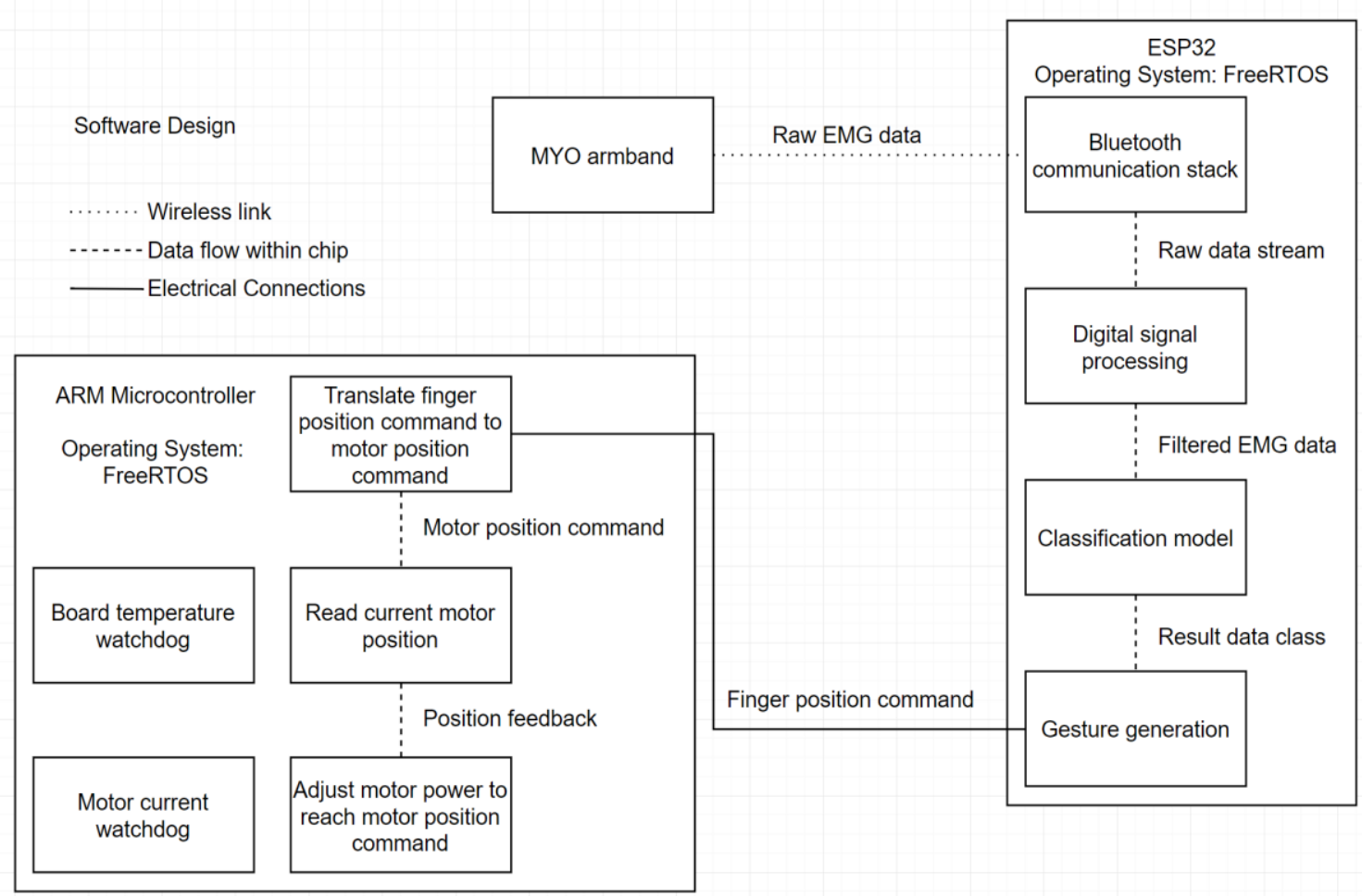

Figure 7. High level open-loop motion command through gesture mapping from EMG signal

In development is a model-based closed-loop control that fits between the gesture generation and motor command signals to create a truly customized user experience for different users and hardware. Grimmer et al. classifies the control of a prosthetic device into either Computational Intrinsic Control (CIC) with little connection to the user, and Interactive Extrinsic Control (IEC) which is based on afferent feedback as in Sparthan's use case.

Using the classical MRAC (Model Reference Adaptive Control) framework, the individualized plant model can be generated through the use of Matlab Systems Identification Toolbox as applied in Simscape import of the unique prosthetic hand CAD model.

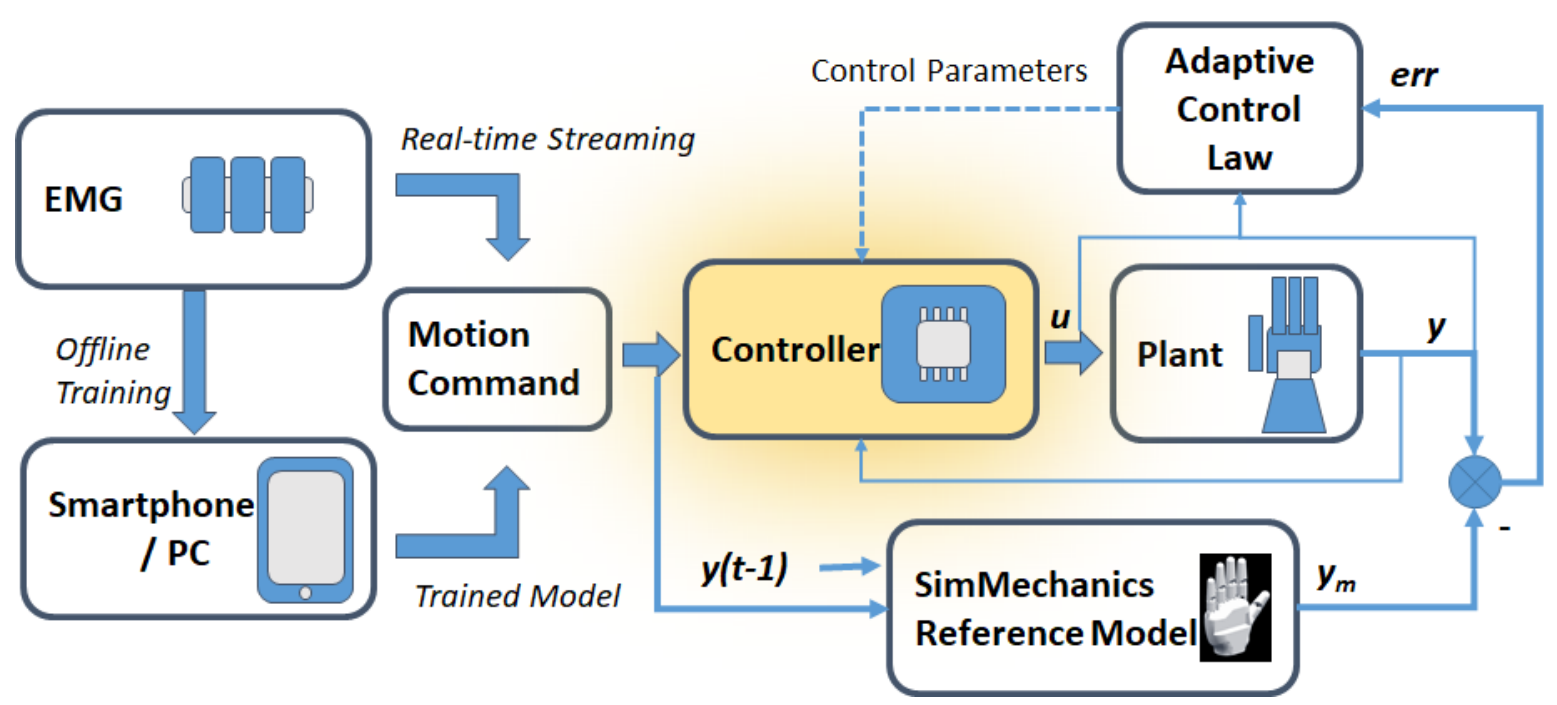

Figure 8. Model-based adaptive control to provide tailored controller design

\subsection{User interface and experience}

A user-friendly app will allow creating a 3D model of the user's body. The images are acquired by a normal smartphone camera and processed to create an accurate 3D model. The custom model will be sent to a 3D printing facility near the user to make delivery even faster. 


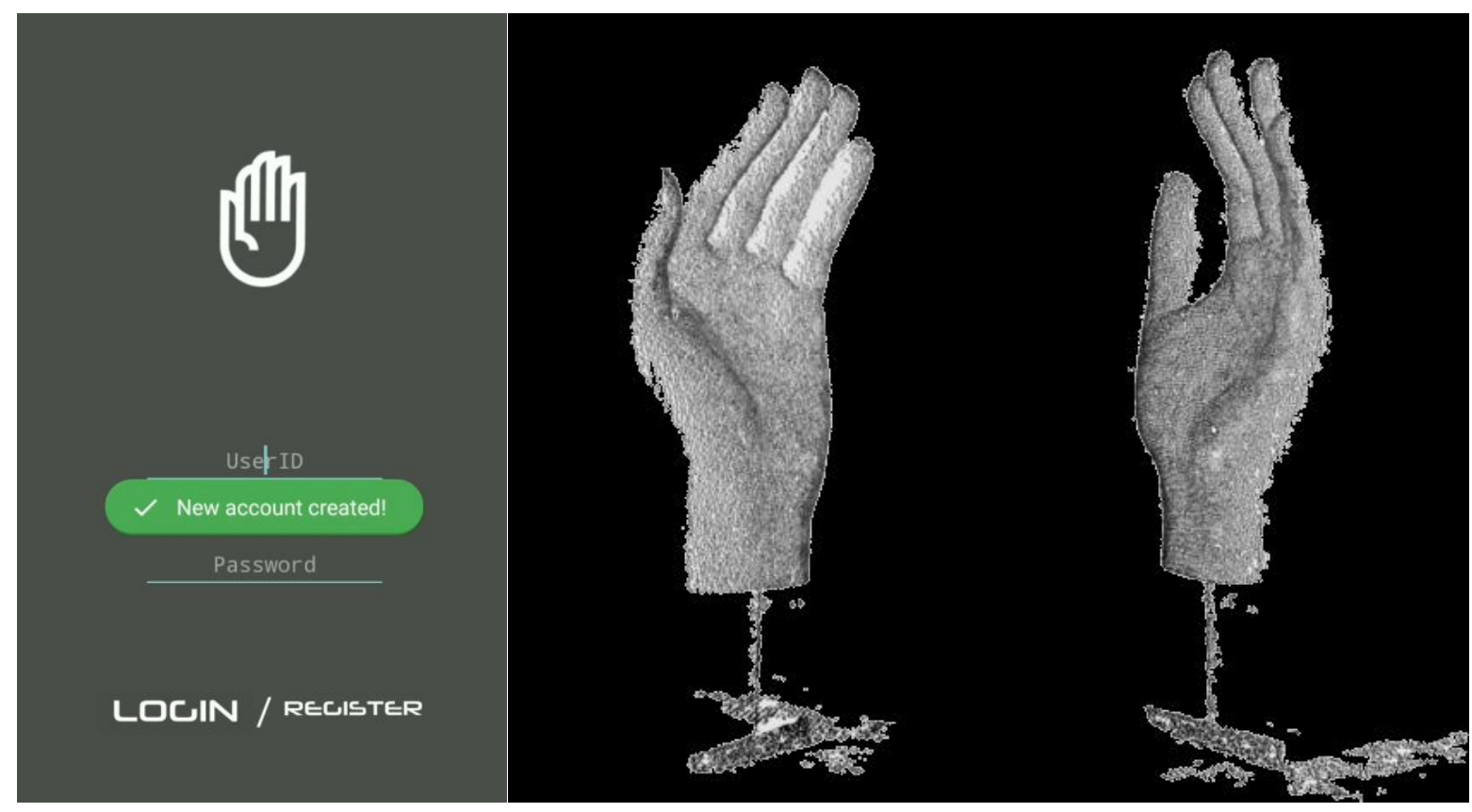

Figure 9. Sparthan App for scanning hand for rehabilitative application

Partnering with makerspaces and schools enables Sparthan to take full advantage of the already existing network of 3D printers. The electronics are designed to be plug-and-play, meaning that once the hand is printed it's just a matter of snapping the module we will ship in place. The goal is to maximize opportunities for end users to be involved in the fitting process via online tools.

\section{CONCLUSIONS AND FUTURE WORKS}

Started with social entrepreneurial ambition, Sparthan employed a combination of lean development process and a series of product design tools (e.g. Kano model and QFD) to arrive within 6 month at a scalable and low-cost solution to advanced prosthesis. Mechanically, the focus was placed on optimizing the housing to hold the motion control board against size, durability and ease of integration requirements. Early evaluations of commercially available EMG sensors resulted in the use of Myoband in the current prototype. The authors are aware of the pending discontinuation of the off-theshelf product; and are now developing own customized EMG sensing array. Finally, with more training data collected through user testing for signal mapping to gesture generation, model-based adaptive controller can be designed and deployed as part of Sparthan's on-boarding experience.

Our solution relies on scalability to ensure effectiveness, for this reason we identified as key partners the 3D printing providers, which can use already existing facilities to print such a low volume application at virtually no additional cost. Providing only the electronics module will lower the initial capital needed for manufacturing, creating a platform on which to build more advanced prosthetics solutions. The key value propositions of the Sparthan solution are:

- Compatibility with existing 3D hands models

- Easy installation procedure for the 3D maker community

- Intuitive control interface for the end user

In 2019, we will focus on introducing the Sparthan kit to beta test with stakeholders; from medical rehabilitation units to prosthetic users. Partnerships with UC Berkeley Million Hands project and local NGOs grant-based social impact deployment are formed to further the project opportunities. The fine tuning of system modelling and adaptive control will be done in tandem to better the overall user on boarding experience. Finally, we also look to employing the mechatronic enabling kit concept to other applications beyond 3D printed hand prosthesis; e.g. motorization of lightweight exoskeleton.

\section{REFERENCES}

Bahari, M., Jaffar, A., Low, C., Jaafar, R., Roese, K. and Yussof, H. (2011), "Design and Development of a Multifingered Prosthetic Hand". International Journal of Social Robotics. Vol. 4,

http://doi.org/10.1007/s12369-011-0133-8. 
Benalcázar, M.E., Jaramillo, A.G., Zea, J.A., Paez, A., and Andaluz, V.H. (2017), "Hand gesture recognition using machine learning and the Myo armband" 25th European Signal Processing Conf. (EUSIPCO), Kos, Greece, 28 Aug - 2 Sep, 2017: pp. 1040-4, http://doi.org/10.23919/EUSIPCO.2017.8081366.

Dalley, S.A., Wiste, T.E., Withrow, T.J. and Goldfarb, M. (2009), "Design of a Multifunctional Anthropomorphic Prosthetic Hand With Extrinsic Actuation," IEEE/ASME Transactions on Mechatronics, Vol. 14 No. 6, pp. 699-706, Dec. 2009. http://doi.org/10.1109/TMECH.2009.2033113

Kontoudis, G. P., Liarokapis, M. V., Zisimatos, A. G., Mavrogiannis, C. I. and Kyriakopoulos, K. J. (2015) "Open-Source, Anthropomorphic, Underactuated Robot Hands with a Selectively Lockable Differential Mechanism: Towards Affordable Prostheses", IEEE/RSJ International Conference on Intelligent Robots and Systems (IROS), Hamburg (Germany), http://doi.org/10.1109/IROS.2015.7354209

ten Kate, J., Smit, G. and Breedveld, P. (2017), “3D-printed upper limb prostheses: a review”, Disability and Rehabilitation: Assistive Technology, Vol. 12 No. 3, pp. 300-314, http://doi.org/10.1080/17483107.2016.1253117

Peerdeman, B., Boere, D., Kallenbergy, L., Stramigioli, S. and Misra, S. (2010), “A Biomechanical Model for the Development of Myoelectric Hand Prosthesis Control Systems" Conference proceedings: Annual International Conference of the IEEE Engineering in Medicine and Biology Society. IEEE Engineering in Medicine and Biology Society. Conference. 2010, pp. 519-23. http://doi.org/10.1109/IEMBS.2010.5626085.

Schmidt, R., Chen, V., Gmeiner, T. and Ratto, M. (2015), “3D-printed prosthetics for the developing world”, SIGGRAPH 2015: Studio (SIGGRAPH '15). ACM, New York, NY, USA, Article 21, 1 pages. https://doi.org/10.1145/2785585.2792535

Tasar, B., Gulten, A. and Yakut, O. (2018), "Modeling, Simulation and Control of Prosthetic Hand using SimMechanics”, International Journal of Current Engineering and Technology, Vol. 8 No. 2, https://doi.org/10.14741/ijcet/v.8.2.2

Visconti, P., Gaetani, F., Zappatore, G.A. and Primiceri, P. (2018), "Technical Features and Functionalities of Myo Armband: An Overview on Related Literature and Advanced Applications of Myoelectric Armbands Mainly Focused on Arm Prostheses”, International Journal on Smart Sensing and Intelligent Systems. Vol 11 No 1, ISSN (Online) 1178-5608, http://doi.org/10.21307/ijssis-2018-005 